\title{
Is local participation always optimal for sustainable action? The costs of consensus-building in Local Agenda 21
}

\author{
Urs Steiner Brandt ${ }^{\mathrm{a}, *}$, Gert Tinggaard Svendsen ${ }^{\mathrm{b}}$ \\ ${ }^{a}$ Department of Environment and Business Economics, University of Southern Denmark, Esbjerg, Denmark \\ ${ }^{\mathrm{b}}$ Department of Political Science and Government, Aarhus University, Denmark
}

\section{A R T I C L E I N F O}

\section{Article history:}

Received 28 September 2012

Received in revised form

28 May 2013

Accepted 15 July 2013

Available online 22 August 2013

\section{Keywords:}

Sustainability

Local Agenda 21

Community participation

Self-organisation

Consensus-building

Free riding

Information gathering

\begin{abstract}
A B S T R A C T
Is local participation always optimal for sustainable action? Here, Local Agenda 21 is a relevant case as it broadly calls for consensus-building among stakeholders. Consensus-building is, however, costly. We show that the costs of making local decisions are likely to rapidly exceed the benefits. Why? Because as the number of participants grows, the more likely it is that the group will include individuals who have an extreme position and are unwilling to make compromises. Thus, the net gain of self-organization should be compared with those of its alternatives, for example voting, market-solutions, or not making any choices at all. Even though the informational value of meetings may be helpful to policy makers, the model shows that it also decreases as the number of participants increase. Overall, the result is a thought provoking scenario for Local Agenda 21 as it highlights the risk of less sustainable action in the future.
\end{abstract}

(c) 2013 Elsevier Ltd. All rights reserved.

\section{Introduction}

One main offspring from Rio 1992 was the UN action plan "Agenda 21". The implementation of Agenda 21 was intended to involve action at international, national, regional, and local levels. Thus, local community participation is the cornerstone in the socalled Local Agenda 21 or "LA21". Here, national and state governments have legislated or advised that local authorities take steps to implement the plan locally (UN, 2009). ${ }^{1}$

Our main research question is whether local participation is always optimal for sustainable action. The fundamental insight of the paper is local participation may, in fact, not always improve decision making on sustainability issues and, in particular, regarding the

\footnotetext{
* Corresponding author.

E-mail addresses: usb@sam.sdu.dk (U.S. Brandt), gts@ps.au.dk (G.T. Svendsen). 1 According to Harvold (2003), different models and approaches have been proposed and applied to facilitate local engagement in the decision making process. Processes might involve stakeholders, interest groups and or may include public involvement. The process might be structured around implementing/solving a particular issue (co-management approach) or oriented broader to consensus building or mutual understanding and information gathering about public preferences (forum approaches).
}

asserted importance of local decision makers' involvement in the process of implementing sustainability issues under LA21.

Thus, theory may challenge the LA21 view by arguing that the costs of self-organization are often ignored and no alternative costs considered. This is a missing link in the literature as previous authors have mainly focused on the benefits from local participation. McKenzie-Mohr (2011) reviews the literature and lists actions that can enhance sustainable behaviour on the local community basis by using marketing tools to encourage positive social change. At least three main advantages from citizen participation in government decision making can be found. First, it is argued that participation leads to a stronger democracy where formulated policies are more realistically grounded in citizen preferences. Second, local participation means higher legitimacy and fewer conflicts so that the public is more likely to accept the tough decisions that government has to come up with. Third, generating new information for decision makers increases the quality of political decisions and boosts efficiency gains (e.g., Rosenström and Kyllöen, 2007; Irvin and Stansbury, 2004).

Our model challenges the second and third point above in the issue of sustainability and agenda 21 . We analyse under which circumstances a voluntary formed group will achieve consensus over providing a public goods (interpreted as a 
sustainable action), and thereby overcome the free riding problem. $^{2}$

Regarding the second point that local participation implies fewer conflicts, our model shows that in a cost-benefit setting, the costs for participants of reaching a consensus might be higher than the benefits to the individuals, implying that participant will not reach a consensus.

This leads us to the third point mentioned above. In this context, chapter 28 of Agenda 21 stipulates that Local officials should consult citizens and community, business and industrial organizations to gather information and build a consensus on sustainable development strategies. This consensus would help them re-shape local programmes, policies, laws and regulations to achieve Agenda 21 objectives. The process of consultation would increase people's awareness of sustainable development issues.

The language calls not for consensus per se but consensus building. We interpret the movements towards consensus as a proxy for the information value that the process generates for the local officials and thereby can characterize the information that the local policy-makers are able to extract from this process. We also derive conditions under which the information content actually falls. This happens in case of a breakdown where participants choose more extreme positions than their initial position. ${ }^{3}$

Overall, the literature does not undertake a more comprehensive cost-benefit analysis, which is strongly needed to grasp the full picture of local participation in sustainable action, defined as any initiative that reduces current resource usage for the sake of producing more resource availability for future generations.

In any case the individuals are motivated to participate if they conceive a net benefit from participation. The starting point is that citizens' willingness to contribute to a public good (or here, willingness to sacrifice consumption to increase the consumption possibilities of future generations) vary from very low to very high (e.g., Ostrom, 2000). However, compared to the classical public good provision, the marginal per capita return (MPCR) (the rate that the contributor receives back from the last contributed unit) is very low. This is so because the benefit from the public good is for the future generation. Second, since the future generation is not yet born, it cannot punish the non-contributor. Several studies of public good provision games show that low MPCR and lack of punishment options are the main reasons for low contribution together with large group sizes (Chaudhuri, 2011; Poulsen, 2009).

The implication is that individual citizens have no or very small incentives to act sustainably. Moreover, group size and social interaction matter when struggling to overcome the collective action problem. ${ }^{4}$ Hence, an obvious way to increase contribution is for citizens to generate the necessary common understanding to overcome the individual incentives of non-contribution. ${ }^{5}$ According to public choice theory, however, it does not follow that perfect consensus, both on the desire for reaching a specific goal and the most efficient means of obtaining it, will always bring about the achievement of a group goal, such as providing sustainable action at

\footnotetext{
${ }^{2}$ Focus here is on local participation, where sustainable behaviour could materialize through neighbourhood initiatives (e.g., parent involvement in local school affairs), influencing workplace social movements (e.g., social networks like Facebook groups) or developing local network enterprises. Relevant issues could be energy savings, local initiatives for greening the economy, buying goods that are produced in a sustainable manner, or even local support for development projects in least developed regions.

${ }^{3}$ We are grateful to an anonymous referee for making this point

4 See e.g., Olson (1965), Ostrom and Ahn (2009), Chaudhuri (2011) and Brandt and Svendsen (2011)

5 Sugden (1984) observed that if sufficiently many participants meet and act in a sustainable way, a tipping point is reached after which the provision of public goods becomes the norm in society.
}

the local level. Mancur Olson invented the modern concept of free riding, defining it this way: “...rational, self-interested individuals will not act to achieve their common or group interests. Those who do not pay for a collective good cannot be excluded from consuming it" (Olson, 1965, 2). This means that economically rational agents may not pursue the collective interest of providing sustainable action at the local level as rational individual behaviour does not lead to rational group behaviour. Even if the total benefits by far exceed the total costs of achieving a common goal, it does not follow that collective action occurs (Svendsen and Svendsen, 2004). Olson's theory of collective action has been called "the central subject of political science" (Ostrom, 1998, 1).

In line with the free rider argument in Olson (1965), we propose yet another reason why contribution from a group tends to fall when an extra participant joins in. The argument is that the more participants, the more likely the group will include individuals who hold extreme positions and are inflexible and not willing to make compromises. ${ }^{6}$ Individual citizens enter the meeting, but their behaviour is guided by cost-benefit considerations. Benefits from participating, broadly speaking, emerge from reaching a common understanding of the level of contribution and partly from the contribution itself. The costs to a citizen arise partly from moving away from his or her preferred position and partly from opportunity costs from participating in the meeting. It is assumed that citizens keep on participating as long as their estimates of costs and benefits from further participation encourage them to do so. Irvin and Stansbury (2004) have argued that public participation may have benefits that outweigh the costs under certain conditions. We, however, add to this literature by introducing a formal group dynamics model to analyse under what conditions benefits outweighs costs of such a policy.

A key issue is the way participants interact in order to approach an acceptable situation for every participant. Gabbey (2007) provides a good description of the group dynamics. The starting point is that a given participant's instantaneous position is subject to change under the influence of three separate forces: (i) the self-bias force, (ii) the group influence force, and (iii) the information flow force. According to Gabbey (2007), a participant's natural preference can often be assumed to be fixed. If a participant's position shifts from a natural preference because of group pressure or new information, he or she will experience a psychological force that resists this change, referred to as self-bias force. The more a person is committed to its natural preferences, the more difficult it becomes to shift the participant away from it. The group influence force is the total force acting to change a given member's position through the influence of the other group members. Such a force is often asymmetric and, among others, depends on relative status, control of resources, expertise and charisma, and personal relationship. Other types of forces are persuasive efforts: respect, reputation or personal integrity, friendship or animosity. The last force is the information flow force, which measures the effect of incoming information of a participant's current position. A key parameter here is the "contextual sensitivity" which measures how a participant responds to new information that challenges his or her initial position.

As in Gabbey (2007), we consider situations where it is not possible to affect peoples' natural preferences. However, participants can be moved away from this position to round-specific positions. The more a person is committed (to his or her own preferred position), the more difficult this process is. Following Coenen (2009),

\footnotetext{
${ }^{6}$ Brandt and Svendsen (2009) provide another analysis where the extreme positions of players affect the outcome of an interaction between fishermen and biologists.
} 
Gabbey (2007), and Irvin and Stansbury (2004), we develop a social interaction model for reaching consensus among local participants in Section 2. In Section 3, we derive the main results regarding conditions for consensus, while the analysis of the information content is done in Section 4. Section 5 gives the conclusion.

\section{A social interaction model for reaching consensus}

The point of departure is that when people interact, they influence each other in ways that make their positions converge. The aim of the "meeting" is to agree upon how many resources the group is willing to allocate to future generations. Essentially, the issue concerns an agreement about the provision of a public good for the future generation. Because of the complexity of group dynamics, we make a number of simplifying assumptions that structure the problem sufficiently, keeping in mind that the model still provides important information about the nature of local participation. The main driving force to engage in the meetings is to reach a consensus: What establishes the right solution is what the group agrees upon. Hence, the right number of resources for future generations is determined by the group's shared understanding of what the group can agree upon. Therefore, the quality of the decision is measured by the probability of reaching consensus.

The fundamental behavioural assumption is that participants' positions converge according to the prescribed group-dynamic interaction. At the end of a round, all participants announce whether or not to continue to the next round. If at least one participant announces that he/she will not continue, the process stops, and the participants are asked whether or not they wish to accept the current position, otherwise the process continues. Finally, the participants are motivated by self-interest, as explained formally below.

Formally, the set of participants is denoted $I=\{1,2, \cdots, n\}$. Let rounds be indexed by $t=0,1,2, \cdots$. Participants are equipped with a fixed initial position, denoted $P_{0}^{i}$, which measures their initial willingness to contribute to the collective and sustainable good. We assume that positions are located between 0 and 1 , where 0 means no contribution and 1 means some well-defined upper limit. In the process of interaction, forces like persuasion, willingness to make compromises, or group pressure push each participant's actual position away from $P_{o}^{i}$ towards $P_{t}^{i}$ in round $t$, which we will comprise in individual cost and benefit functions.

The process of convergence of the participants' current positions in ways that respect the group dynamics needs to be formulated. A simplifying assumption throughout the analysis is that the probability of consensus is determined solely by the difference between the two extreme positions ( $P_{t}^{\max }$ is denoted the highest and $P_{t}^{\min }$ the lowest contribution in round $t$ ) around the average position of the participants: $P_{t}^{\text {dif }}=P_{t}^{\max }-P_{t}^{\min }$. Let the probability of consensus in round $t$ be $\operatorname{prob}_{t}^{\text {cons }}$. We assume that $\partial \operatorname{prob}_{t}^{\text {cons }} / \partial P_{t}^{\text {dif }}<0$, that is, the smaller the distance between the positions of the two most extreme positions around the average positions of the participants, the larger the probability of consensus. In what follows, the following normalization will be convenient: $\operatorname{prob}_{t}^{\text {cons }}=1$ if $P_{t}^{\text {dif }}=0$ and $\operatorname{prob}_{t}^{\text {cons }}=0$ if $P_{t}^{\text {dif }}=1$. We also define the probability of a breakdown in a given period as $\operatorname{prob}_{t}^{\mathrm{bd}}=1-\operatorname{prob}_{t}^{\text {cons }}$.

We here present the functional linear relationships between $\operatorname{prob}_{t}^{\text {cons }}$ and $P_{t}^{\text {dif }}$ that we apply in our simulations. ${ }^{7}$ In this particular case, $\operatorname{prob}_{t}^{\mathrm{bd}}=P_{t}^{\text {dif }}$, the probability of a breakdown is equal to the difference in the two opposite extreme positions.

We can also derive the expected contribution in a given round (if this round will be the final round) as $E\left(\operatorname{Con}_{t}\right)=\operatorname{prob}_{t}^{\text {cons }} \cdot \sum_{i=1}^{I} P_{t}^{i}$. The average contribution in a given round is $E\left(\operatorname{Con}_{t}^{A}\right)=E\left(\operatorname{Con}_{t}\right) / n$.

\footnotetext{
${ }^{7}$ We could also apply a non-linear relationship, e.g., a logistic function.
}

In our presentation, we focus on changes in positions in a round. $\Delta P_{t}^{i}=\left(P_{t}^{i}-P_{t-\text { ini }}^{i}\right)$ is defined as the change in position in round $t$ from the initial position $P_{t-i n i}^{i}$ to the announced position $P_{t}^{i} \cdot P_{t}^{j+}$ is defined as the initial positions in round $t$ of participants placed to the right of the average of the initial positions of this period, while $P_{t}^{j-}$ are the initial positions to the left of the average of round $t$. (The average position in a given round is defined as $P_{t}^{A}=1 / I \cdot \sum_{i=1}^{I} P_{t-\text { ini }}^{i}$ ).

Since this might turn out to be a rather complicated process, we propose that in a given period, participants are grouped into those with positions lower than period (initial) average $\left(j^{-}\right)$and those with positions above the average $\left(j^{+}\right)$. To ease notation, we however suppress the "+"and "-“" signs and always consider that a $\Delta P_{t}$ is a movement towards the average contribution.

Our fundamental behavioural assumption is that participants behave in a tit-for-tat manner. We formulate this as a friendliness assumption: In each round $t$, a friendly move is given by a movement towards the average position in that round. A friendly move is reciprocated by all other participants by a subsequent move towards the average. This is shown in Fig. 1. A movement of participant $i$ in round $t$ towards the average is followed by a movement of participant $j$ towards the average as well.

Further, we need to explain the dynamics towards consensus in such a group by specifying the net benefit for the participants from moving towards consensus and the dynamics describing how individual participants react on the other participants' moves. We should keep in mind the fundamental trade-off a participant faces when moving away from his or her preferred position. It is costly to this participant, but on the other hand, it also provides benefits: The expected benefit is that such a move will have a positive influence on the other participants' willingness to move away towards the initiating participant's position.

The assumed behaviour of the participants is to maximize their net benefit in each period, by making an optimal choice of $\Delta P_{t}^{i}$. They are, however, also concerned about consensus and the total size of the contribution. This behaviour implies that a participant shows willingness to participate as long as total net benefit from the meeting remains non-negative. Therefore, the participants are willing to continue even though, on the margin, continuation yields a loss to this participant.

Finally, to put some more structure on the process, we require that

Assumption A1. if $\Delta P_{t}^{\text {dif }}=0$, then the process stops in this round.

Assumption A2. In case of breakdown, the participants remain at the position they had in the last round.

Note that in case of breakdown, A2 requires that participants remain at the end position. In Section 4 we consider other possible reactions among participants in case of breakdown.

The net benefit function for participant $i$ from changing his or her position in period $t$ by $\Delta P_{t}^{i}$, which we consider in its general form, is given by ${ }^{8}$ :

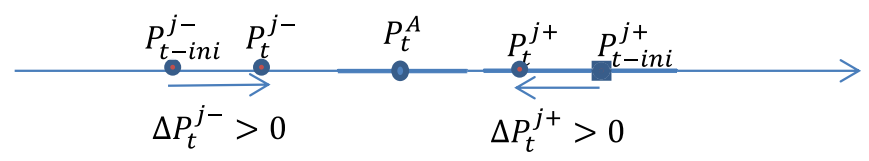

Fig. 1. The move of position in a two-participant situation.

\footnotetext{
8 Since we only consider changes in position in a given period, the arguments in the $B$ and $C$ functions are all in changes as well (with the exception of $P_{t-i n i}^{i}$ ). In (1), we only include the effect of a participant's own change. As will be seen in the twoparticipant example below, the other participants' actions are captured be the reaction functions that link the net benefit functions together.
} 
$\mathrm{NB}_{t}^{i}\left(\Delta P_{t}^{i}\right)=B_{t}^{i}\left(\Delta p_{t}^{\mathrm{cons}}\left(\Delta P_{t}^{i}\right) ; \operatorname{Con}_{t}\left(\Delta P_{t}^{i}\right)\right)-c_{t}^{i}\left(P_{t-\mathrm{ini}}^{i}, \Delta P_{t}^{i}\right)-\mathrm{FC}^{i}$

where we always define changes as movements towards the average contribution. Assumed is:

$\frac{\partial B_{t}^{i}}{\partial \Delta P_{t}^{\text {cons }}}>0$

$\frac{\partial B_{t}^{i}}{\partial \Delta \operatorname{Con}_{t}}>0$

A participant can benefit in two ways from moving their own position towards the average position. The first part of the benefit function captures that such a move increases the probability of consensus. The second part captures that the participants also benefit from the increase in the overall expected contributions, which is positively affected by a participants' move towards the average position. The expected change in the contribution level is given by:

$\Delta \operatorname{Con}_{t}\left(\Delta P_{t}^{i}\right)=\left[\Delta p_{t}^{\text {cons }}\left(\Delta P_{t}^{\mathrm{dif}}\left(\Delta P_{t}^{i}\right)\right) \cdot \sum_{j=1}^{I} P_{t}^{i}\left(\Delta P_{t}^{i}\right)\right]$

and consists of two elements. The first shows that the change in $P_{t}^{i}$ affects the probability of consensus (through the friendliness assumption), and the second part shows that such a change also changes the position of the other participants.

$\Delta P_{t}^{\text {dif }}=\Delta P_{t}^{\text {dif }}\left(\Delta P_{t}^{i}\right)$

The friendliness assumption implies that:

$$
\begin{aligned}
& \frac{\mathrm{d} \Delta P_{t}^{\text {dif }}}{\mathrm{d} \Delta P_{t}^{i}}>0 \\
& \frac{\mathrm{d} \operatorname{Con}_{t}\left(\Delta P_{t}^{i}\right)}{\mathrm{d} \Delta P_{t}^{i}}= \frac{\mathrm{d} P_{t}^{\text {cons }}}{\mathrm{d} \Delta P_{t}^{\mathrm{dif}}} \cdot \frac{\mathrm{d} \Delta P_{t}^{\mathrm{dif}}}{\mathrm{d} \Delta P_{t}^{i}} \cdot \sum_{j=1}^{I} P_{t}^{i}\left(\Delta P_{t}^{i}\right)+P_{t}^{\text {cons }} . \\
&\left(1+\sum_{j \neq i} \frac{\mathrm{d} P_{t}^{i}\left(\Delta P_{t}^{i}\right)}{\mathrm{d} \Delta P_{t}^{i}}\right)>0
\end{aligned}
$$

$\frac{\mathrm{d} B_{t}^{1}\left(\Delta P_{t}^{1}\right)}{\mathrm{d} \Delta P_{t}^{1}}=b^{11} \frac{\mathrm{d} \Delta p_{t}^{\text {cons }}\left(\Delta P_{t}^{1}\right)}{\mathrm{d} \Delta P_{t}^{1}}+b^{12}\left[\frac{\mathrm{d} \Delta p_{t}^{\text {cons }}\left(\Delta P_{t}^{1}\right)}{\mathrm{d} \Delta P_{t}^{1}}\left(\Delta P_{t}^{1}+\Delta P_{t}^{2}\left(\Delta P_{t}^{1}\right)\right)+\Delta p_{t}^{\text {cons }}\left(\Delta P_{t}^{1}\right) \cdot\left(1+\frac{\mathrm{d} P \Delta_{t}^{2}\left(\Delta P_{t}^{1}\right)}{\mathrm{d} \Delta P_{t}^{1}}\right)\right]$

Hence, since we always define changes as movements towards the average contribution, such a movement always implies that the total contribution increases.

It follows from (1a), (1b), (4) and (5) that:

$\frac{\mathrm{d} B_{t}^{i}\left(\Delta P_{t}^{i}\right)}{\mathrm{d} \Delta P_{t}^{i}}=\frac{\partial B_{t}^{i}}{\partial P_{t}^{\text {cons }}} \cdot \frac{\mathrm{d} P_{t}^{\text {cons }}}{\mathrm{d} \Delta P_{t}^{i}}+\frac{\partial B_{t}^{i}}{\partial \operatorname{Con}_{t}} \cdot \frac{\mathrm{dCon}_{t}}{\mathrm{~d} \Delta P_{t}^{i}}>0$

Regarding the cost function used in (1), $c_{t}^{i}=c_{t}^{i}\left(P_{t-\mathrm{ini}}^{i}, \Delta P_{t}^{i}\right)$. Essentially, this cost function is intended to capture that the further away a participant is from the initial position, the more costly an

\footnotetext{
${ }^{9}$ When making the simulation, we will, however, sidestep this assumption slightly.
}

additional move away from this position will be. ${ }^{9}$ Formally, this is described by:

$\frac{\partial^{2} c_{t}^{i}}{\partial \Delta P_{t}^{i} \partial P_{t-\text { ini }}^{i}}>0$

This implies that (1) exhibits a fundamental asymmetry between the way benefits and costs are experienced. In any period, where a participant is not at the initial position, it will be costly to this participant. On the other hand, benefits are only experienced by additional changes in position compared to the previous period which is in the line of Gabbey (2007). The implication is that if status quo occurs in a given period, and a participant has moved away from the initial position (when arriving at this period) will result in a negative net benefit in that period.

Regarding opportunity costs, participation is obviously timeconsuming and represents real costs. We assume that these costs are the same in each round for a participant, but they are not necessarily identical for different participants.

We apply a linear net benefit function of the following type:

$$
\begin{aligned}
\mathrm{NB}_{t}^{i}\left(\Delta P_{t}^{i}\right)= & b^{i 1} \cdot \Delta p_{t}^{\text {cons }}\left(\Delta P_{t}^{i}\right) \\
& +b^{i 2} \cdot\left[\Delta p_{t}^{\text {cons }}\left(\partial \Delta P_{t}^{i}\right) \cdot\left(\partial \Delta P_{t}^{i}+\sum_{j \neq i} \Delta P_{t}^{j}\left(\Delta P_{t}^{i}\right)\right)\right] \\
& -c^{i 1} \cdot\left(\left|P_{0}^{i}-P_{t-1}^{i}\right|\right)-c^{i 2} \cdot\left(\Delta P_{t}^{i}\right)^{2}
\end{aligned}
$$

Now, we are in the position to make more specific interferences. We first look at a two-participant linear example, with participants 1 and 2 . Focussing first on the benefit function and participant 1 , we have that

$$
\begin{aligned}
B_{t}^{1}\left(\Delta p_{t}^{\text {cons }}\left(\Delta P_{t}^{1}\right) ; \operatorname{Con}_{t}\left(\Delta P_{t}^{1}\right)\right)= & b^{11} \cdot \Delta p_{t}^{\text {cons }}\left(\Delta P_{t}^{1}\right) \\
+b^{12} \cdot & {\left[\Delta p_{t}^{\text {cons }}\left(\Delta P_{t}^{1}\right)\right.} \\
& \left.\left(\Delta P_{t}^{1}+\Delta P_{t}^{j}\left(\Delta P_{t}^{i}\right)\right)\right]
\end{aligned}
$$

Differentiating with respect to $\Delta P_{t}^{1}$ yields:
Consider first $\mathrm{d} \Delta p_{t}^{\text {cons }}\left(\Delta P_{t}^{1}\right) / \mathrm{d} \Delta P_{t}^{1}$ and let $P_{0}^{2}>P_{0}^{1}$.

We have that:

$$
\Delta P_{t}^{\text {dif }}\left(\Delta P_{t}^{1}\right)=P_{t-\text { ini }}^{2}-\Delta P_{t}^{2}\left(\Delta P_{t}^{1}\right)-\left[P_{t-\text { ini }}^{1}+\Delta P_{t}^{1}\right]
$$

The tit for tat behaviour is embodied in the reaction functions. We use the following reaction function:

$\Delta P_{t}^{1}\left(\Delta P_{t}^{2}\right)=\gamma^{12} \cdot \Delta P_{t}^{2}$

$\Delta P_{t}^{2}=\gamma^{21} \cdot \Delta P_{t}^{1}$

We let $0<\gamma^{i j}<1$, such that the reaction function implies that a movement towards the average of another participant is reciprocated by a movement in the direction of the average (but less than 1 to 1 ). We assume linearity, but $\gamma^{12} \neq \gamma^{21}$ is a possibility. 
Inserting $\Delta P_{t}^{2}\left(\Delta P_{t}^{1}\right)=\gamma^{21} \cdot \Delta P_{t}^{1}$ gives:

$\Delta P_{t}^{\text {dif }}\left(\Delta P_{t}^{1}\right)=P_{t-\text { ini }}^{2}-\gamma^{21} \cdot \Delta P_{t}^{1}-\left[P_{t-\text { ini }}^{1}+\Delta P_{t}^{1}\right]$

Rearranging

$\Delta P_{t}^{\mathrm{dif}}\left(\Delta P_{t}^{1}\right)=P_{t-1}^{\mathrm{dif}}-\left(1+\gamma^{21}\right) \cdot \Delta P_{t}^{1}$

$\frac{\mathrm{d} \Delta p_{t}^{\text {dif }}\left(\Delta P_{t}^{1}\right)}{\mathrm{d} \Delta P_{t}^{1}}=-\left(1+\gamma^{21}\right)$

Linearity is assumed, such that

$\Delta p_{t}^{\text {cons }}\left(\Delta P_{t}^{1}\right)=1-\Delta P_{t}^{\text {dif }}\left(\Delta P_{t}^{1}\right)$

And it follows that:

$\frac{\mathrm{d} \Delta p_{t}^{\text {cons }}\left(\Delta P_{t}^{i}\right)}{\mathrm{d} \Delta P_{t}^{i}}=\left(1+\gamma^{21}\right)$

Inserting all this into the differentiated benefit function yields:

$$
\begin{aligned}
\frac{\mathrm{d} B_{t}^{1}\left(\Delta P_{t}^{1}\right)}{\mathrm{d} \Delta P_{t}^{1}}= & \left(1+\gamma^{21}\right)\left[b^{11}+b^{12}\left(\Delta P_{t}^{1}+\gamma^{21}\right)\right] \\
& +\left(1+\gamma^{21}\right) \cdot \Delta P_{t}^{1} \cdot\left(1+\gamma^{21}\right)
\end{aligned}
$$

The cost function is given by:

$c_{t}^{i}\left(P_{t-\mathrm{ini}}^{i}, \Delta P_{t}^{i}\right)=c^{i 1} \cdot\left(\left|P_{0}^{i}-P_{t-1}^{i}\right|\right)-c^{i 2} \cdot\left(\Delta P_{t}^{i}\right)^{2}$

The first part of the cost function captures that cost increases, the further away the participant is positioned from his or her initial position (assuming a linear relationship). The second part captures that any movement away from the position of the last period is costly and increasing with distance. This second cost type only occurs in one period.

We are now in the position to derive the convergence process by using the above specification. Since participants are assumed to optimize, we can find the first order condition for optimality. Differentiating the net benefit function with $\Delta P_{t}^{i}$ yields:

$$
\begin{aligned}
\frac{\mathrm{dNB}_{t}^{1}\left(\Delta P_{t}^{1}\right)}{\mathrm{d} \Delta P_{t}^{1}}= & \left(1+\gamma^{21}\right)\left[b^{11}+b^{12}\left(\Delta P_{t}^{1}+\gamma^{21}\right)\right]+\left(1+\gamma^{21}\right) . \\
& \Delta P_{t}^{1} \cdot\left(1+\gamma^{21}\right)-2 c^{i 2} \Delta P_{t}^{i}=0
\end{aligned}
$$

From this, we find the optimal change in position in any round as a function of the parameters:

$$
\Delta P_{t}^{1}=\frac{b^{12} \cdot \gamma^{21}}{\frac{2 c^{12}}{\left(1+\gamma^{21}\right)}-b^{12}-\left(1+\gamma^{21}\right)}
$$

The values of $\gamma^{i j}, c^{i}, b^{i}$ determine the speed of convergence and the net benefit in each round. For a given $\gamma^{i j}$, the larger the ratio $b^{i}$ / $c^{i}$, the more likely that consensus is reached. Fixed costs do not enter the convergence process but are relevant for the decision to participate. The consequence is that $\mathrm{FC}^{i}$ is simply an additional cost borne by each participant in each additional round and will therefore reduce each period NB and thereby the likelihood of consensus.

In the example given in Fig. 2, the process stops in round 7. Here, the probability of consensus is 0.89 , and the expected contribution

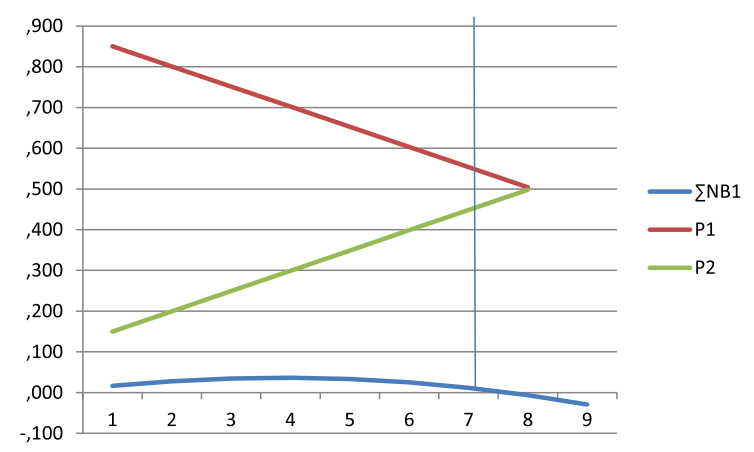

Note: Parameter values: $\gamma^{12}=\gamma^{21}=0.1, c^{11}=c^{21}=0.1, c^{12}=c^{22}=10, b^{11}=b 1^{21}=0.7, b^{12}=b^{22}=1, F C^{1}=F C^{2}=0.01$

Fig. 2. Convergence for a two-participation model.

is also 0.89 . Note that if the participants continue another round, the expected contribution will increase to 0.98. This will not happen, however, since the participants would then receive a net loss from participating. This example shows the fundamental trade-off that the participants face.

From (9), several results can be deduced. First of all, an increase in $\gamma^{12}$ increases the amount that a participant is willing to move in each round, which shows the reciprocal nature of the process. Second, an increase in $c^{12}$ reduces the movement and an increase in $b^{11}$ increases the movement towards the average position. In general terms:

$\frac{\mathrm{d} \Delta P_{t}^{i}}{\mathrm{~d} b^{i 1}}>0, \frac{\mathrm{d} \Delta P_{t}^{i}}{\mathrm{~d} c^{i 2}}<0, \frac{\mathrm{d} \Delta P_{t}^{i}}{\mathrm{~d} \gamma^{j i}}<0$

Regarding the length of the arrangement (i.e., the number of rounds), the probability of consensus, and the expected contribution, we can make some changes in the parameters to obtain further insight in these effects, which are presented in Table 1. Focussing on the expected contribution, it should be realized that any change in the parameters implying a slowing of the convergence process also implies a lower expected contribution due to the presence of the fixed opportunity cost and the reciprocal nature of the response functions.

\section{Increasing the number of participants}

This section is devoted to the question of the effects of increasing the number of participants. To keep the analysis tractable, we will not make formal derivation of the participants' response function in this section, but instead assume that the participants follow a "rule of thumb" dynamics described as follows:

$\Delta P_{t}^{i}=\gamma_{t}^{i} \cdot\left(\frac{b^{i}}{c^{i}}\right) \cdot\left(P_{t-1}-P_{t-1}^{A}\right)$

where we have that

$b^{i}=b^{i 1}+b^{i 2}$

$c^{i}=c^{i 1}+c^{i 2}$

$P_{t}^{A}=\left(\frac{1}{n}\right) \cdot \sum P_{t-\mathrm{ini}}^{i}$

$\gamma_{t}^{i}=\alpha^{i} \cdot \frac{\sum_{j} \gamma_{t-1}^{i}}{n}, \forall \alpha^{i}>0, t>0$

Expression (9a) captures the essence of (9). The response parameter $\gamma_{t}^{i}$ has, however, been changed. This function captures 
Table 1

The two-participants example.

\begin{tabular}{lllll}
\hline Parameter value & $\begin{array}{l}\text { Last round before } \\
\text { breakdown }\end{array}$ & $P_{t}^{\text {dif }}$ & $\begin{array}{l}\text { Probability } \\
\text { of consensus }\end{array}$ & $\begin{array}{l}\text { Expected } \\
\text { contribution }\end{array}$ \\
\hline Fig. 2 & 7 & 0.11 & 0.89 & 0.89 \\
$\left(\gamma^{21} \uparrow\right)$ & 6 & 0.06 & 0.94 & 0.94 \\
$\left(c^{21} \uparrow\right)$ & 8 & 0.43 & 0.57 & 0.57 \\
$\left(\mathrm{FC}^{1} \uparrow\right)$ & 5 & 0.3 & 0.7 & 0.7 \\
\hline
\end{tabular}

the essential behaviour observed in experiments with public goods provision games (Ostrom and Ahn, 2009). Each player (participant) starts out with a positive move (i.e., a move towards the average position) depending on his/her initial type (the $\gamma_{0}^{i}$ 's). Hereafter, players to a more or less extent act as reciprocal players. If sufficiently many contribute relatively small amounts, the other players also respond by contributing less. Here, the observation from Ostrom (2000) is that some people continue to contribute a great deal while others quickly begin to contribute very little. This effect is captured by the $\alpha^{i}$ s. An $\alpha^{i}<1$ implies that this participant tends to respond less and less (unless the other participants increase their response very much) while a $\alpha^{i}>1$ implies that this participant tends to respond more and more (unless the other participants reduce their response very much).

The participants are still assumed to have the net benefit function as prescribed by (8), and their behaviour is still to continue to as long as the total net benefit from participating remains nonnegative. Moreover, the process again stops if a participant does not want to continue.

A specific simulation result is presented in Fig. 3, where we have five participants, and Fig. 3 shows the convergence path together with the "binding" net benefit function.

One issue we have not touched upon so far is the number of participants. To analyse this in the frame of our model so far, we can interpret $\alpha^{i}$ as a measure of the willingness to make compromises. Now consider that for each participant, $\alpha^{i}$ is drawn (independently) from a given probability distribution function (pdf). For example, assume that the $\alpha^{i}$ 's are normally distribution according to:

$\alpha^{i} \sim N\left(\bar{\alpha}, \sigma_{\alpha}\right)$

Consider next that we wish to find the probability that at least one participant out of a total of seven in a group, randomly chosen,

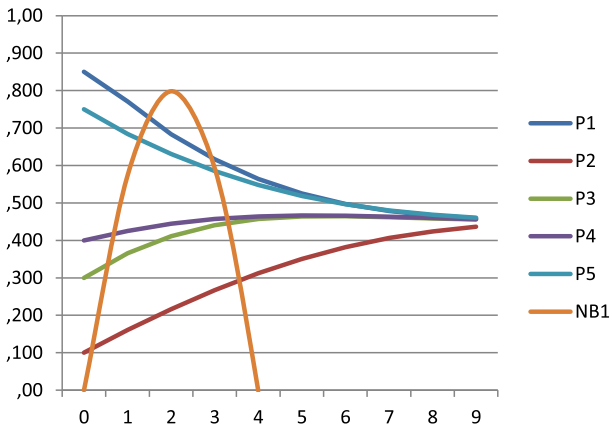

Note: P1 to P5 denote participants.

\begin{tabular}{|c|c|c|c|c|c|c|c|c|c|c|c|}
\hline & & & 15 & & & & & & & & \\
\hline$I$ & 1 & 2 & 3 & 4 & 5 & $I$ & 1 & 2 & 3 & 4 & 5 \\
\hline $\mathrm{P}_{0}^{\mathrm{i}}$ & 0.85 & 0.10 & 0.5 & 0.4 & 0.75 & $c^{i 2}$ & 0.1 & 0.1 & 0.1 & 0.1 & 0.1 \\
\hline $\mathrm{b}^{\mathrm{i} 1}$ & 4 & 3,7 & 5 & 5 & 5 & $\mathrm{FC}^{\mathrm{i}}$ & 0.01 & 0.01 & 0.01 & 0.01 & 0.01 \\
\hline $\mathrm{b}^{\mathrm{i} 2}$ & 1 & 1 & 1 & 1 & 1 & $\gamma_{0}^{\mathrm{i}}$ & 0.2 & 0.2 & 0.2 & 0.2 & 0.2 \\
\hline$c^{\mathrm{i} 1}$ & 4 & 4 & 4 & 4 & 4 & $\alpha^{i}$ & 1.1 & 0.7 & 1.3 & 1.3 & 1 \\
\hline
\end{tabular}

Fig. 3. Example with 5 participants. has $\alpha^{i}<\alpha^{\prime}$, where $\alpha^{\prime}$ is a value to the left of the pdf (e.g., 10\%). This probability can be calculated using the binomial distribution, where the number of trails is the number of participants and the probability is the probability of obtaining a $\alpha^{i}<\alpha^{\prime}$ in a single trail. Letting $n=7, p=0.1$ and $x=0$, we have that:

$P(x, n, p)=\left(\begin{array}{l}n \\ k\end{array}\right) p^{n} p^{n-x}$

$P(0,7,0.1)=0.48$

Increasing $n$ to, say, $n=15$ reduces the probability to 0.23 . Now there is a probability of 0.77 that a participant with little willingness to seek compromises is included.

Using (9a) and (9b), it follows that $\mathrm{d} \Delta P_{t}^{i} / \mathrm{d} \alpha^{i}>0$. Therefore, the larger the number of participants, the larger the probability of including individuals that only change position slowly because of their unwillingness to make compromises. This, however, might only be a problem for the process if this participant is decisive for the probability of consensus, for example, if this participant has an extreme position.

The second parameter that determines the change in the probability of consensus, and thereby also the number of rounds needed to arrive at a consensus, is the initial position of the participants. Consider again that this parameter is drawn independently for each participant from a probability distribution function (pdf) and that for each participant, $P_{0}^{i}$ is also drawn (independently) from a given pdf. We assume that the $P_{0}^{i}$ 's are normally distributed according to:

$P_{0}^{i} \sim N\left(\bar{P}_{0}, \sigma_{p_{0}}\right)$

We can again argue that the probability of including a participant with an 'extreme' position increases as the number of participants increase. The worst case for the ability to reach consensus is when a participant is included that both holds an extreme position and little willingness to move at the same time.

If we consider that both parameters are to be independent (and non-correlated), it follows that as the number of participants increase, the probability of including an extreme-position, nonconsensus type of participant increases.

The more participants included, on average, the more the speed of convergence is reduced. The consequence is that the average contribution in each round is reduced. Thus, the net benefit for the participants is reduced in each round (all else equal). ${ }^{10}$ It follows from (8) that

$\frac{\mathrm{dNB}_{t}^{i}}{\mathrm{~d} p_{t}^{\text {cons }}}>0$

As a result, the contribution per participant is expected to diminish as the number of participants increase. To illustrate this result, consider that changing $\alpha^{1}$ to, say, 0.1 but keeping the average $\alpha$ at 1 implies that the probability of consensus is only 0.32 , so that the total contribution is reduced to $56 \%$.

Our final finding here is that since the participants meet in order to come to an agreement about their position with respect to "producing" sustainable behaviour, the inclusion of more participants reduces the potential achievement of that group in this respect.

As already mentioned in the introduction, participation and participants can take a variety of forms. In many real world

\footnotetext{
10 It should be noted that as $n$ increases, the effect of changes in one participant $a^{i}$ or $\gamma^{i}$ has a diminishing effect on the reaction of the other participants. Therefore, "second-order" effects can be neglected for high $n$.
} 
situations (on sustainability issues), participants are not necessarily randomly drawn from a population. The reason for this is that we would expect that in the self-selection process, the most extreme positions enter first (having either most at stake or the highest interest in this issue). ${ }^{11}$ As a consequence, the probability of participants holding extreme positions need not rise in expectation with the number of participants.

There is, however, no reason to expect that very ideological groups (or participants holding extreme positions) are more willing to make compromises than individuals without special interests. E.g., Irvin and Stansbury (2004) find that if a participation model that is heavy influenced by opposing interest groups, often end up making worse policy decisions. In this case, the participants with the smallest alpha $i$ will also enter first. Therefore, the probability that less compromising participants are included need not increase in this particular case.

On the other hand, in this extreme case (where those with most extreme positions and least willingness to change positions are those who first will enter such groups), increasing the number of participants will essentially have no effect on the process. In this special case, we need to modify our result to: The probability of consensus never increases for an increase in $n$. Only in the special case where those with most extreme positions and lower willingness to make compromises enter the process first, the probability of consensus is unchanged as $n$ increases.

\section{The value of the consensus process for the policy maker}

Until now, the purpose of the local participation process has been to overcome the free riding incentives in a "voluntary contribution to a public good for future generations" problem. Now consider that the consensus building process is a part of a larger policy process. As described in the introduction, in the context of Agenda 21, local officials should consult citizens and community, business and industrial organizations to gather information and build a consensus on sustainable development strategies.

It is, therefore, very important to consider what can happen in case of a breakdown of the process. In Section 2, the assumption was that the participants even in case of a breakdown were reacting positive towards the process and remained at the end position, which were closer to average than their initial position. This we can denote the optimistic scenario. Here, the process would increase people's awareness of sustainable development issues even in the case of a breakdown.

However, other outcomes are possible. E.g., the neutral case, where the participants would fall back to their initial position, and finally the pessimistic case, where participants due to disappointments in the process end up choosing more extreme positions than their initial position.

The pessimistic scenario has been described in Sunstein (2002), who defines group polarization as a situation where members of a group move towards more extreme points, where extreme is solely internal, referring to the members' initial (pre-) position. ${ }^{12}$

\footnotetext{
11 Often, participation takes one of two forms: individuals are either invited to join the process because they represent groups with specific ideologies or participants self-select into the process (which, given some cost of participating, implies that those with extreme positions will be most likely to participate in the process). As an example of a group with specific ideologies can be mentioned New Zealand's Land and Water Forum (2013) with the Environmental Defence Society at the table with Federated Farmers [Thanks to an anonymous referee for making this valuable point].

12 According to Sunstein (2002), a mechanism that underlies polarization is peoples' desire to maintain reputation and their self-conception. The effect is reinforced in the case where the group consists of equally opposed subgroups.
}

In our model, the participants basically were reciprocal in the sense that moves toward average were acknowledged. However, people tend to be "two-sided" reciprocal, such that "bad" behaviour is punished. Rabin (1993) suggests that the sign of our sentiments is conditional in that the same people who are altruistic to other altruistic people are also motivated to hurt those who hurt them. Note that in our setting, the only behaviour through which participants can reciprocate it through changes in position. In this way, discouraged participants can by either blaming the "opposite side" for lack of flexibility or to maintain reputation and their selfconception move to more extreme positions in case of a breakdown.

How then to determine the value of the information that the officials will receive from engaging in the consensus building process in the framework of the model specified in Section 2? We model the information content is by the variance in the data. That is, the variance of the end positions of the process. If consensus is reached, information is perfect, but if no consensus is reached, the larger the variance in the positions, the less valuable is the information that comes out of the consensus building process. To capture this, consider that the expected value of the information that the officials gather from the process can be written as:

$E V[I]=p^{\text {Cons }} \cdot V\left(I^{C}\right)+\left(1-p^{\text {Cons }}\right) \cdot V\left(I^{\mathrm{NC}}\right)-V\left(I^{\mathrm{INI}}\right)$

Here $V(I)$ is the value function and $I$ an index for information. We let $\mathrm{d} V(I) / \mathrm{d} I>0$ and for simplicity normalize $I[0,1]$. Note that $I^{C}<I^{\mathrm{INI}}$, the information content in consensus is larger than in the initial situation. We need to determine $V(I)$, information in the nonconsensus case. As already noted, information content is measured by the variance of the end positions. Letting the vector of end position be $\boldsymbol{p}^{\text {end }}$ and the be $\operatorname{var}\left(\boldsymbol{p}^{\text {end }}\right)$. Information depends on the variance, and overall we let: $I=I\left(\operatorname{var}\left(\boldsymbol{p}^{\text {end }}\right)\right)$, with $\mathrm{d} I / \operatorname{dvar}\left(\boldsymbol{p}^{\text {end }}\right)<0$. Larger variance provides less information. In consensus, $I$ is maximized (variance is zero) while in case of two equally sized opposing groping each at extreme points ( 0 and 1$)$ yields the least information value.

As an example, given the hypothesis that participants in the case of a breakdown fall back to the initial position, and assuming that the NB still is a described by [1], we can calculate the value of the process when the convergence slows down in a two-participants setting. Take the values specified in Table 1 . And consider a specific linear value function: $V(I)=I$ and $I=1-4\left(\right.$ variance $\left.\left(P_{i}^{1} ; P_{i}^{2}\right)\right)$. The $I$ function simply states that given consensus, $I=1$ and given minimal information (position 1 and 0 ) then $I=0$.

For the parameter values specified in Table $1, E V[I]=0.93$, while reducing the gamma to 0.04 . Implies $E V[I]=0.87$, a reduction of the information value of $6.4 \%$.

More generally, in our model, for the same initial position, the information value for all situations will fall,

$$
\frac{\partial E V(I)}{\partial C} \leq 0
$$

$\frac{\partial E V(I)}{\partial \gamma} \geq 0$

11a is valid for all cost types in the model. As costs increase, the value of process is reduces due to 1 ) smaller probability of consensus and 2) larger positional difference in case of a breakdown.

In general, if the probability of consensus is small enough, then information value turn out to be negative, in particular in the pessimistic case. Small changes in the parameter values compared 
to those in Fig. 2 yield a negative information value for the pessimistic case. ${ }^{13}$

Now we can make the more general observation. Increasing $n$ reduces the expected value for the policy makers as long as all participants in case of breakdown fall back to their initial positions. This follows directly from the results in Section 3. More participants imply a larger probability of slow convergence and therefore consensus will be less likely. More participants also imply a larger likelihood to include extreme positions, which implies both lower probability of consensus and larger divergence if no consensus is reached. Finally, negative information (in the sense that less information is provided than without the group meetings) cannot be ruled out, in particular when participants after a breakdown move to more extreme positions than their initial position.

\section{Conclusion}

Is local participation always optimal for sustainable action? Selforganization and decentralized decision making may overcome free-riding problems and enhance the quality of decisions, that is, small local communities may solve sustainability problems better compared to the situation where formal rules are exogenously imposed by a third-party authority. When people meet on equal terms, exchange views, discuss, and interact, a potential for making decisions may arise that has qualities that regular voting does not hold.

Overcoming the free-rider-problem is, however, costly. Our social interaction model argued that a cost-benefit analysis must be applied. Most prominently, the model showed that the more participants, the more likely it is that the group will include individuals who have an extreme position and are not willing to make compromises.

We show that the costs of making local decisions are likely to rapidly exceed the benefits. Why? Because as the number of participants grows, the more likely it is that the group will include individuals who have an extreme position and are unwilling to make compromises. Thus, the net gain of self-organization should be compared with those of its alternatives, for example voting, market-solutions, or not making any choices at all.

Local Agenda 21 was a relevant case as it broadly calls for consensus-building among stakeholders. Also in this case, a certain point existed beyond which the costs of self-organization would outweigh the potential benefits. In spite of the fact that the informational value of meetings could be helpful to policy makers, the model showed that it also decreased as the number of participants increased. In perspective, the net gain of self-organization and consensus-building may have been overestimated so far in the literature. Local participation is not always optimal for sustainable action.

\section{Acknowledgements}

An earlier version of this paper was presented at the ISEE conference in Rio de Janeiro, June 16-19, 2012. We thank the other participants and two anonymous referees for helpful comments.

\section{References}

Brandt, U.S., Svendsen, G.T., 2009. Trawling for subsidies. Journal of European Public Policy 16 (7), 1012-1029.

Brandt, U.S., Svendsen, G.T., 2011. A project-based system for including farmers in the EU ETS. Journal of Environmental Management 92 (4), 1121-1127.

Chaudhuri, A., 2011. Sustaining cooperation is laboratory public goods experiments: a selective survey of the literature. Experimental Economics 14, 47-83.

Coenen, F.J.M., 2009. Introduction, chapter 1. In: Coenen, Frans H.J.M. (Ed.), Public Participation and Better Environmental Decisions: the Promise and Limits of Participatory Processes for the Quality of Environmentally Related Decisionmaking. Springer.

Gabbey, M., 2007. The effects of nonlinear interactions and network structure in small group opinion dynamics. Physica A: Statistical Mechanics and Its Applications 378, 118-126.

Harvold, K.A., 2003. Consensus or conflict? Experiences with local agenda 21 forums in Norway. Local Government Studies 29, 117-135.

Irvin, R.A., Stansbury, J., 2004. Citizen participation in decision making: is it worth the effort? Public Administrative Review 64, 55-65.

Land and Water Forum, 2013. New Zealand. http://www.landandwater.org.nz/Site/ About_Us/Forum_Members.aspx\#H126742-2 (accessed 09.04.13.).

McKenzie-Mohr, D., 2011. Fostering Sustainable Behavior: an Introduction to Community-based Social Marketing, third ed. New Society Publishers.

Olson, M., 1965. The Logic of Collective Action. Cambridge University Press, Cambridge.

Ostrom, E., 1998. A behavioral approach to the rational choice theory of collective action. American Political Review 92 (1), 1-22.

Ostrom, E., 2000. Collective actions and the evolution of social norms. Journal of Economic Perspective 14, 137-158.

Ostrom, E., Ahn, T.K., 2009. The meaning of social capital and its link to collective action. In: Svendsen, G.T., Svendsen, G.L.H. (Eds.), Handbook of Social Capital. The Troika of Sociology, Political Science and Economics. Edward Elgar, Cheltenham, pp. 17-35.

Poulsen, A., 2009. Cooperation: evidence from experiments. In: Svendsen, G.T., Svendsen, G.L.H. (Eds.), Handbook of Social Capital: the Troika of Sociology. Political Science and Economics. Edward Elgar, Cheltenham, pp. 36-56.

Rabin, M., 1993. Incorporating fairness into game theory and economics. American Economic Review 83, 1281-1302.

Rosenström, U., Kyllöen, S., 2007. Impacts of a participatory approach to developing national level sustainable development indicators in Finland. Journal of Environmental Management 84, 282-296.

Sunstein, C.R., 2002. The law of group polarization. Journal of Political Philosophy 10 (2), 175-195.

Sugden, R., 1984. Reciprocity: the supply of public goods through voluntary contributions. Economic Journal 94, 772-787.

Svendsen, G.L.H., Svendsen, G.T., 2004. The Creation and Destruction of Social Capital. Edward Elgar Publishing, Cheltenham, UK.

UN, 2009. Agenda 21. http://www.un.org/esa/dsd/agenda21/ (accessed 07.05.12.).

\footnotetext{
13 Note: Parameter values: $\gamma^{12}=\gamma^{21}=0.04, c^{11}=c^{21}=0.2, c^{12}=c^{22}=10$, $b^{11}=b^{21}=0.6, b^{12}=b^{22}=1, \mathrm{FC}^{1}=\mathrm{FC}^{2}=0.01$ yields $E V(I)=-0.01$ in the pessimistic situation, while in the optimistic situation $E V(I)=0.35$. Note that highest possible value is 1 .
} 